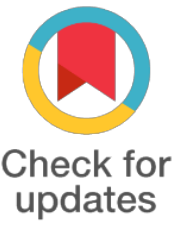

*For correspondence:

nguyendaihai@iams.vast.vn

Competing interests: The authors declare that no competing interests exist.

Received: 2017-05-15

Accepted: 2017-06-18

Published: 2017-09-05

Copyright The Author(s) 2017. This article is published with open access by BioMedPress (BMP).

This article is distributed under the terms of the Creative Commons Attribution License (CC-BY 4.0) which permits any use, distribution, and reproduction in any medium, provided the original author(s) and the source are credited.

\section{Gelatin-Poly (Ethylene Glycol) Methyl Ether- Functionalized Porous Nanosilica for Controlled Doxorubicin Delivery}

Uyen Vy Vo 1, 2, 3 , Tuong Vi Tran 1, 2, Ngoc Tram Nguyen Thi 1, 2, 4, Anh Khoa Nguyen 1, 2, 4, Bao Yen To Thi 1, 2, 5, Cuu Khoa nguyen 1,2, Dai Hai Nguyen 1, 2 *

1 Graduate University of Science and Technology, Vietnam Academy of Science and Technology, 18 Hoang Quoc Viet, Cay Giay, Hanoi 100000, Vietnam

2 Institute of Applied Materials Science, Vietnam Academy of Science and Technology

01 TL29, District 12, HCM

\section{Abstract}

Porous nanosilica (PNS) has been attracting a growing attention in fabrication of carriers for drug delivery system (DDS). However, unmodified PNS-based nanocarriers exhibit the initial burst release of encapsulated bioactive molecules, which may limit their potential clinical applications. In this report, the surface of PNS was conjugated with gelatin-poly (ethylene glycol) methyl ether (GEL-mPEG) to form a core-shell structure PNS-GEL-mPEG for doxorubicin (DOX) delivery. The conjugated PNS carriers were found to be spherical in shape with diameter range of approximately 55-85 $\mathrm{nm}$ as compared with their parentally PNS (55-67 nm). The PNS-GEL-mPEG nanoparticles showed their ability to effectively encapsulate DOX for controlled release. In detail, DOX was efficiently loaded into the PNS-GEL-mPEG to form DOX-loaded nanocarriers (DOX@PNS-GEL-mPEG) with high loading efficiency (79.7\%). The release of DOX from DOX@PNSGEL-mPEG was prolonged and controlled up to $96 \mathrm{~h}$ in phosphate buffered saline (PBS, pH 7.4, 37 $\mathrm{oC})$ without any initial burst release. These results demonstrated that this PNS-GEL-mPEG can be a potential candidate for controlled DDS with high loading capacity in cancer therapy.

\section{Keywords}

porous nanosilica, gelatin, polyethylene glycol, drug delivery system, cancer therapy

\author{
Funding
}

References 